\title{
nature
}

28 August 2003 Volume 424 Issue no 6952

\section{Drugs are for beauty too}

Like it or not, many people want to improve their looks with the help of biology, and pharmaceutical companies look set to help them. Regulatory bodies need to catch up.

$\mathrm{T}$ hey want to be pert, they want to be taut, they want glossy manes into their 60s. And they want balms, dyes and depilatories that will help them achieve it. Some might dismiss it as vanity, but society's increasing preoccupation with looks is fuelling a booming business in cosmetic drugs, or 'cosmeceuticals', worth $\$ 3.4$ billion last year in the United States alone.

Cosmeceuticals lurk in the shadowy ground between drugs and cosmetics. Allergan's Botox, which flattens furrowed foreheads, is one example. Merck's Propecia for balding pates is another, as are off-the-shelf skin creams with active biological ingredients.

Those who add a prescription cosmeceutical to their morning routine can have real hope of seeing results. But the enticing skincare aisles of your local drugstore tell a different story: dermatologists confess that some $90 \%$ of ingredients in anti-ageing creams are little more than overpriced petroleum jelly. Why, when you slather on jelly for wrinkles, do you need to smear on a healthy dose of scepticism?

Cosmeceutical manufacturers must shoulder much of the blame, for trying to sidestep drug regulations. The US Food and Drug Administration (FDA) defines drugs as agents intended for use in the diagnosis, cure, mitigation, treatment or prevention of disease, or that affect the structure or function of the body. Cosmetics escape the rigorous trials demanded of a drug because they are assumed only to alter our appearance. But the system falls down for cosmeceuticals, because it is the manufacturers - not the FDA - who decide whether a product is classed as drug or cosmetic. A cream that claims to cure eczema is a drug; if it claims to promote healthy skin, it is a cosmetic. This bizarre situation means that, although some cosmetics companies have excellent R\&D arms, many are dissuaded from finding genuinely active ingredients, or advertising their properties if they do, for fear of having to undergo expensive drug trials. This also fuels the pseudo-science that is used to hype cosmetics.

This state of affairs must change. Cosmeceutical manufacturers should show that their ingredients genuinely work, or find some that do. The FDA should revise its outdated regulations to enforce this, so that all biologically active ingredients, whether drugs or cosmetics, have to prove their worth. Consumers are helpless to tell fact from fiction in today's non-prescription creams, so there is a market for prescription drugs with proven cosmetic powers. A few pharmaceutical and cosmetics giants, including Pfizer and L'Oreal, are embarking on research to fill this niche. Some people might argue that pharmaceutical companies should concentrate on curing deadly diseases, not feeding society's body paranoia. But if there is money in the beauty parlour, they are likely to pursue it (see page 990).

Perhaps a more thorny dilemma will face doctors who have to decide whether to prescribe cosmetic drugs to patients who demand them for wrinkles or baldness. As with any non-essential therapy, national or private health insurers can exclude them from coverage. But doctors and medical ethicists must consider whether they have the right to refuse a prescription to patients willing to pay.

The dilemmas thrown up by cosmeceuticals will become more pressing as biologists reveal the secrets behind wilting skin and barren follicles - and the molecules that might revamp them. Our attitudes are outdated. Consumers should demand more from their \$20-apot unguents. And when 'real' cosmeceuticals are created, doctors, industry and regulators should work together to ensure that they are effective and accessible.

\section{Fetal trials need public funds}

The outcomes of trials of fetal-cell transplants highlight the importance of public access.

ast week, neurologists finally published the long-awaited results of a clinical trial that has been controversial since its outset (see page 987). The study was the second of two funded by the US National Institutes of Health (NIH) to look at whether fetal-cell transplants could ease the symptoms of Parkinson's disease. The controversy continues, as neuroscientists argue over what the results mean, but the bottom line is that public funding in this emerging field ensured that the trials were done in the most open, rigorous, ethical and safe way possible. Policy-makers and funding agencies should keep this lesson in mind, especially in a political climate that is increasingly hostile towards other areas of research, such as work on human embryonic stem cells.

The Parkinson's trials were first opposed because they used tissue from aborted fetuses. The US government could not fund such trials because of restrictions preventing scientists from using federal funding for work on fetal tissue. But after then President Bill Clinton lifted the restriction on federal funding for fetal-tissue research, scientists began two NIH-funded trials of the technique.

In 2001, a publication on the first Parkinson's trial showed that some patients had developed involuntary muscle movements called 'dyskinesias'. The second trial found the same side-effects. But these were publicly funded trials, and the researchers were free to discuss their results in journals and at meetings, so scientists now have some idea about what caused the dyskinesias. More importantly, they have publicly available data to help them evaluate their ideas.

This is a very different situation from some privately funded trials, such as an Alzheimer's-vaccine trial that was stopped last year when some patients developed life-threatening brain inflammation in a study sponsored by a company called Elan. The side-effect was reported in a company press release, not at a scientific meeting. The scientific community, short of data, is still trying to work out what went wrong.

This is a crucial lesson for countries such as the United States that are trying to decide how much to get involved in work on human embryonic stem cells. Whether or not governments fund such work, investors will eventually fund private trials themselves. If policymakers want to ensure that the data from such trials do not disappear into a black hole, and that trials are done as safely as possible, it should err on the side of more, not less, funding for the work. 